\title{
Game chromatic number of Cartesian product graphs*
}

\author{
T. Bartnicki ${ }^{\dagger}$ \\ Faculty of Mathematics, Computer Science, \\ and Econometrics \\ University of Zielona Góra \\ 65-516 Zielona Góra, Poland \\ T.Bartnicki@wmie.uz.zgora.pl
}

\author{
B. Brešar \\ University of Maribor, FEECS \\ Smetanova 17, 2000 Maribor, Slovenia \\ bostjan.bresar@uni-mb.si
}

\section{J. Grytczuk}

Theoretical Computer Science Department Faculty of Mathematics and Computer Science Jagiellonian University

30-387 Kraków, Poland

Grytczuk@tcs.uj.edu.pl

\section{Z. Miechowicz}

Faculty of Mathematics, Computer Science, and Econometrics

University of Zielona Góra

65-516 Zielona Góra, Poland

barta@poczta.onet.pl

\section{Kovše}

Faculty of Natural Sciences and Mathematics

University of Maribor

Koroška cesta 160, 2000 Maribor, Slovenia

matjaz.kovse@gmail.com

\author{
I. Peterin ${ }^{\ddagger}$ \\ University of Maribor, FEECS \\ Smetanova 17, 2000 Maribor, Slovenia \\ iztok.peterin@uni-mb.si
}

Submitted: Apr 9, 2007; Accepted: May 5, 2008; Published: May 12, 2008

Mathematics Subject Classification: 05C15, 91A46

\begin{abstract}
The game chromatic number $\chi_{g}$ is considered for the Cartesian product $G \square H$ of two graphs $G$ and $H$. Exact values of $\chi_{g}\left(K_{2} \square H\right)$ are determined when $H$ is a path, a cycle, or a complete graph. By using a newly introduced "game of combinations" we show that the game chromatic number is not bounded in the class of Cartesian products of two complete bipartite graphs. This result implies that the game chromatic number $\chi_{g}(G \square H)$ is not bounded from above by a function of game chromatic numbers of graphs $G$ and $H$. An analogous result is derived for the game coloring number of the Cartesian product of graphs.
\end{abstract}

*The paper was started during a meeting in Zielona Góra.

${ }^{\dagger}$ Research supported by a PhD grant from Polish Ministry of Science and Higher Education N201 212833.

${ }^{\ddagger}$ Supported by the Ministry of Higher Education, Science and Technology of Slovenia under the grant P1-0297. The author is also with the Institute of Mathematics, Physics and Mechanics, Jadranska 19, 1000 Ljubljana. 


\section{Introduction}

Various (two-player) games on graphs have been considered, cf. [9]. Among the most intensively studied is the coloring game, whose corresponding graph invariant is called the game chromatic number of a graph, denoted $\chi_{g}(G)$. One of the central questions related to this parameter is concerned with its behavior in graph classes: is there an upper bound for the game chromatic number of graphs from a certain class of graphs $\mathcal{G}$, and if so, determine it as sharply as possible. Let $\chi_{g}(\mathcal{G})=\sup \left\{\chi_{g}(G): G \in \mathcal{G}\right\}$. For the class of planar graphs $\mathcal{P}$ it is known that $8 \leq \chi_{g}(\mathcal{P}) \leq 17$ [13, 18], for the class of outerplanar graphs $\mathcal{O P}$ we have $6 \leq \chi_{g}(\mathcal{O P}) \leq 7[10]$, and for $k$-trees $\mathcal{K} \mathcal{T}$ we have $\chi_{g}(\mathcal{K T})=3 k+2$ for $k \geq 2[16,17]$. For a recent survey see [1] and the references therein.

Let us recall the rules of the game. Players color the vertices of a finite graph $G$, choosing from the set of colors $\{1,2, \ldots, k\}$. The first player (Alice) is aiming that all vertices of $G$ are colored, and the second player (Bob) is trying to prevent this. They alternate turns with Alice starting the game, and the only rule they must obey is to color a vertex with a color different from all the colors that appeared in its neighborhood (at the time of the coloring). If all the vertices of the graph are colored, Alice wins, otherwise Bob wins (that is, at a certain state of the game there appears an uncolored vertex whose neighborhood contains all colors). The smallest $k$ such that Alice has a winning strategy on $G$ using $k$ colors is called the game chromatic number of $G$. The obvious bounds for the game chromatic number are $\chi(G) \leq \chi_{g}(G) \leq \Delta+1$ where $\Delta$ denotes the maximum degree of vertices in $G$.

It is natural to study games on Cartesian product graphs $\mathcal{C} P$; several real-world games are played on grids, which are simple examples of Cartesian products (i.e. products of paths). Since the graphs $G$ and $H$ in the Cartesian product $G \square H$ are arbitrary, $\chi_{g}(\mathcal{C P})$ is not finite. However, one could perhaps expect that $\chi_{g}(G \square H)$ be bounded by some function of $\chi_{g}(G)$ and $\chi_{g}(H)$ which holds for many graph invariants. We show in this paper that even this is not true. In addition a similar result is derived for another wellknown graph game - the marking game.

In the next section we obtain some exact results for special families of Cartesian product graphs, notably for several classes of prisms. In the third section we prove our main result that the game chromatic number is not bounded above by a constant in the class of Cartesian products of two complete bipartite graphs. (Note that $\chi_{g}\left(K_{n, n}\right)=3$ for every $n \geq 2$, so the result implies there is no function $f$ such that $\chi_{g}(G \square H) \leq f\left(\chi_{g}(G), \chi_{g}(H)\right)$ for all graphs $G$ and $H$.) For making the proof of this result more transparent, we introduce the so-called game of combinations which could also be of independent interest. At the end of this section we present some families of Cartesian products of graphs in which the game chromatic number is bounded by a constant, in particular this holds for products of two planar graphs. In Section 4, we first consider the marking game, and derive from a result in [14] that the game coloring number $\operatorname{col}_{g}\left(K_{1, n} \square K_{1, m}\right)$ of the product of two stars can be arbitrarily large. In the rest of this section we discuss some other variations of games on Cartesian products of graphs and hypergraphs. 


\section{Exact values}

We begin by recalling the definition of the Cartesian product of graphs. Let $G$ and $H$ be graphs. The Cartesian product $G \square H$ is the graph with vertex set $V(G) \times V(H)$ where two vertices $\left(u_{1}, v_{1}\right)$ and $\left(u_{2}, v_{2}\right)$ are adjacent if and only if either $u_{1}=u_{2}$ and $v_{1} v_{2} \in E(H)$ or $v_{1}=v_{2}$ and $u_{1} u_{2} \in E(G)$. Graphs $G$ and $H$ are called factor graphs of the Cartesian product $G \square H$. Note that the Cartesian product is commutative and associative with $K_{1}$ as the unit. We say that a connected graph $G$ is in the class $\mathcal{C P}$ if $G$ is isomorphic to the Cartesian product of two non trivial graphs (that is, both different from $K_{1}$ ). For a $v \in V(H)$ the subgraph $G_{v}$ of $G \square H$, induced by $\{(u, v): u \in V(G)\}$ is called a $G$ fiber. Analogously we define $H$-fibers. Reader is referred to [11] for other invariants and properties of product graphs.

Finding the game chromatic number of a graph is in principle a hard problem. In this section we prove exact results of this invariant in some simple Cartesian graph products, i.e. the product of an arbitrary path (cycle, complete graph) by an edge.

Proposition 1. Let $n$ be a positive integer. Then

$$
\chi_{g}\left(K_{2} \square P_{n}\right)= \begin{cases}2 & : \text { for } n=1, \\ 3 & : \text { for } 2 \leq n \leq 3, \\ 4 & : \text { for } n \geq 4 .\end{cases}
$$

Proof. The result is clear for $n=1$ where we have $K_{2} \square P_{1}=K_{2}$. For $n=2$ we have $K_{2} \square P_{2}=C_{4}$ and again everything is clear. For $n=3, K_{2} \square P_{3}$ has two vertices of degree 3 and after the second move of Alice both vertices are colored. Since all remaining vertices have degree 2 , the result is clear also for $n=3$.

Assume $n \geq 4$. Denote the vertices of the two fibers of $P_{n}$ with $v_{1}, v_{2}, \ldots, v_{n}$, and $v_{1}^{\prime}, v_{2}^{\prime}, \ldots, v_{n}^{\prime}$. Suppose that only three colors $\{1,2,3\}$ are available. By symmetry there are only two different cases for Alice's first move. If Alice starts in a vertex of degree 3 say $v_{2}$ with color 1 , Bob responds with color 2 on the vertex $v_{3}^{\prime}$. After Alice's next move, at least one of $v_{3}$ and $v_{2}^{\prime}$ remains uncolored. Thus if $v_{3}$ remains uncolored, Bob colors $v_{4}$ with 3 and $v_{3}$ cannot be colored anymore (with these three colors). Otherwise $v_{2}^{\prime}$ remains uncolored and Bob colors $v_{1}^{\prime}$ with color 3 and $v_{2}^{\prime}$ cannot be colored anymore.

Suppose Alice starts in a vertex of degree 2, say $v_{1}$, with color 1 . Then Bob colors $v_{3}^{\prime}$ with 1. Note that Bob can force Alice to be the first to color a vertex from the set $P=\left\{v_{1}^{\prime}, v_{2}^{\prime}, v_{2}, v_{3}\right\}$ because there is even number of vertices $v_{i}$ and $v_{i}^{\prime}$ for $i \geq 4$, and it is Alice's turn. Also the color 1 cannot be used on the vertices of $P$ anymore. Vertices of $P$ induce a path on four vertices. Since $\chi_{g}\left(P_{4}\right)=3$, and only colors 2 and 3 may be used in $P$, we infer that Bob wins the game. By the trivial upper bound, the proof is complete.

Proposition 2. For an integer $n \geq 3, \chi_{g}\left(K_{2} \square C_{n}\right)=4$.

Proof. Denote the vertices of one fiber of $C_{n}$ with $v_{1}, v_{2}, \ldots, v_{n}$, and with $v_{1}^{\prime}, v_{2}^{\prime}, \ldots, v_{n}^{\prime}$, the corresponding vertices of the second fiber. Suppose that only three colors $\{1,2,3\}$ are 
available. By symmetry there is only one possibility for Alice's first move, say $v_{2}$ with color 1. For $n \geq 5$ Bob responds on $v_{3}^{\prime}$ with 2 and Alice cannot deal with threats on both $v_{3}$ and $v_{2}^{\prime}$, so Bob forces fourth color in his next move. For $n=4$ this strategy does not work, since Alice can color $v_{1}$ with 3 in her second move. But $K_{2} \square C_{4}=Q_{3}=K_{4,4}-M$ ( $M$-perfect matching), hence $\chi_{g}\left(K_{2} \square C_{4}\right)=4$ as well. For $n=3$ Bob responds on $v_{3}^{\prime}$ with 1 , what forces Alice to use a new color and Bob easily wins the game. By the trivial upper bound, the proof is complete.

Proposition 3. For a positive integer $n, \chi_{g}\left(K_{2} \square K_{n}\right)=n+1$.

Proof. For $n=1,2$ the result is clear. We describe a winning strategy for Bob with $n$ colors for $n \geq 3$. In his first $n-2$ moves Bob applies the following rules: (1) he always colors a vertex in the opposite fiber of $K_{n}$ with the same color to the color used previously by Alice; (2) if possible he colors a vertex whose unique neighbor in the opposite $K_{n}$-fiber has been already colored. According to condition (1) of this strategy Alice is forced to use a new color in each her move, so after $n-2$ moves exactly $n-2$ colors will be used. Condition (2) guarantees that after $n-2$ moves of each player all four uncolored vertices induce connected subgraph (either path $P_{4}$ or cycle $C_{4}$ ). Moreover Alice has to start the game on one of the four remaining vertices with only two last remaining colors. In both cases $\left(P_{4}\right.$ or $\left.C_{4}\right)$ Bob wins the game by coloring the eligible vertex with last color. By the trivial upper bound, the proof is complete.

By these results one might think that $\chi_{g}\left(K_{2} \square G\right)=\chi_{g}(G)+1$ in general. Unfortunately this is not so as the following example shows. Let $G$ be obtained from the triangle where two leaves are attached to every vertex. By symmetry Alice can start in a vertex of degree one or four. It is easy to see that Bob can force fourth color no matter where Alice starts. However $\chi_{g}\left(K_{2} \square G\right)=4$ as well. To see this one needs to use a tedious case by case analysis which is left to the reader.

Very often it is not easy to find exact values for $\chi_{g}(G)$ even for relatively small graphs $G$. For instance we have not found the answer for the simplest Cartesian product graphs - the hypercubes $Q_{n}$, which are the products of $n$ copies of $K_{2}$. It is easy to see that $\chi_{g}\left(Q_{n}\right)=n+1$ for $n \in\{1,2,3\}$. The same holds for $n=4$ and we ask whether this holds in general.

Question 1. Is it true that for a positive integer $n$, we have $\chi_{g}\left(Q_{n}\right)=n+1$ ?

An additional difficulty with this invariant is that is not hereditary in general. There are examples of spanning subgraphs with the game chromatic number arbitrarily larger than in their original graphs (e.g. complete bipartite graphs minus a perfect matching as subgraphs of complete bipartite graphs). Nevertheless, we strongly suspect that for any graphs $G$ and $H$ we have $\chi_{g}(G \square H) \geq \max \left\{\chi_{g}(G), \chi_{g}(H)\right\}$. 


\section{The game of combinations and upper bounds for the game chromatic number of Cartesian products}

Before presenting the main result we introduce a new game called "the game of combinations". It may be of independent interest, though we are introducing it mainly for the purposes of proving the main theorem. We note that this game can be regarded as a special case of the well-known Ramsey game, cf. [3, 4].

Let $[n]=\{1, \ldots, n\}$ and let $\mathcal{F}$ be a set whose elements are subsets of $[n]$ with $\lceil n / 2\rceil$ elements (that is, $\lceil n / 2\rceil$-combinations from $[n]$ ). Note that $\mathcal{F}$ may be also empty and it can contain at most $\left(\begin{array}{c}n \\ {[n / 2\rceil}\end{array}\right)$ elements. The game of combinations is always played with respect to some set $\mathcal{F}$ that is known in advance and which we call the set of forbidden combinations.

The game is played by two players who alternate turns. At each move a player picks an element from $[n]$ which has not already been chosen before by one of the two players. At the end both players obtain a combination (a subset of numbers from $[n]$ ) - the first player ends with a $\lceil n / 2\rceil$-combination $C_{1}$, and the second one with a $\lfloor n / 2\rfloor$-combination $C_{2}$. Note that $\left\{C_{1}, C_{2}\right\}$ is a partition of $[n]$.

The first player who starts the game is called the avoider, and his goal is to avoid all combinations from $\mathcal{F}$. The avoider wins the game if the combination $C_{1}$ of the numbers he picked is different from all forbidden combinations (in symbols $C_{1} \notin \mathcal{F}$ ). The second player, called the forcer, wants to force the avoider that the combination chosen by the avoider is from $\mathcal{F}$, that is, the forcer wins if $C_{1} \in \mathcal{F}$. From the definition of the game it follows that there always exists a winning strategy for exactly one of the players. It is clear that $\mathcal{F}=\emptyset$ implies that the avoider has a winning strategy (more precisely, any sequence of moves yields a victory for the avoider). On the other hand $|\mathcal{F}|=\left(\begin{array}{c}n \\ \lceil n / 2\rceil\end{array}\right)$ implies that the second player automatically wins.

Question 2. Let $n$ be an arbitrary number. Suppose the set of forbidden combinations $\mathcal{F}_{0}$ is empty. What is the largest number $k$ of consecutive games $\mathcal{G}_{0}, \ldots, \mathcal{G}_{k}$ where the first player has a winning strategy for all of them and the forbidden set of combinations $\mathcal{F}_{i}$ for the game $\mathcal{G}_{i}$ consists of all $\lceil n / 2\rceil$-combinations of the avoider appearing in games played before the current game?

The problem above is a natural one, if we assume that both players are using optimal strategies. However, even if the forcer plays without thinking, the resulting number $k$ of games that are played is not greater than $\left(\begin{array}{c}n \\ {[n / 2\rceil}\end{array}\right)$. This obvious fact will be implicitly used in the proof of the main theorem.

We will also consider two variations of the game of combinations, where exactly one of the players may skip/pass moves. To make the definitions meaningful, we assume that the game stops when either the avoider picks a $\lceil n / 2\rceil$-combination or the forcer picks a $\lfloor n / 2\rfloor$-combination, and the eventual integers that have not been picked are automatically given to the other player (which has not picked the presumed number of integers). In the first variation, when it is the avoider's turn he either picks an integer or he chooses to 
do nothing and it is forcer's turn again (the avoider may skip one or several moves). We call this variation avoider skip game, or shortly $A S$ game of combinations. Analogously we define the variation where the forcer is allowed to skip moves which we call forcer skip game of combinations (FS game for short).

Lemma 1. If the avoider has a winning strategy in the game of combinations with respect to $\mathcal{F}$, then he also has a winning strategy in the FS game of combinations with respect to $\mathcal{F}$.

Proof. By $x_{1}, y_{1}, x_{2}, y_{2}, \ldots$ we denote the sequence of integers that are picked during a game of combinations (hence $x$-s are moves of the avoider, and $y$-s are moves of the forcer). Since the avoider has a winning strategy, he can choose his moves in such a way that the set of integers $\left\{x_{1}, \ldots, x_{k}\right\}, k=\lceil n / 2\rceil$, picked by him will not be in $\mathcal{F}$.

Now consider the FS game. We may assume that the forcer chooses to pass at least one move. Each time the forcer passes a move, the avoider marks an unpicked (and unmarked) integer, and imagines that the forcer's move consisted of picking this integer. Also, if the forcer picks a marked integer (chosen in some previous steps by the avoider) then the avoider chooses another unpicked and unmarked vertex and marks it. Hence two games are played at the same time - the real game in which some moves of the forcer (denoted by $y_{i}$ ) are missing, and the game, imagined by the avoider which is ordinary game of combinations. In the imagined game, the avoider plays according to a winning strategy which he has by assumption. Hence in the sequence of moves obtained by the imagined game every second move (i.e. $y_{i}, i=1, \ldots, k, k=\lfloor n / 2\rfloor$ ) is played by the forcer. Note that the sequence in the real game is obtained from $S=x_{1}, y_{1}, x_{2}, y_{2} \ldots$ by deleting some $y$-s that indicate the moves that were passed by the forcer and by moving some of these $y$-s in the sequence to the right, which indicates that the forcer picked a (previously) marked vertex. Since the game stops when there are $\lceil n / 2\rceil$ integers chosen by the avoider, we infer that the resulting combination $C_{1}$ of the avoider is the same in both games. Hence $C_{1} \notin \mathcal{F}$.

By using similar arguments one can easily prove also the following lemma.

Lemma 2. If the forcer has a winning strategy in the game of combinations with respect to $\mathcal{F}$, then he also has also a winning strategy in the AS game of combinations with respect to $\mathcal{F}$.

We return to the problem of upper bounds for the game chromatic number of Cartesian products. It is well-known and easy to see that $\chi_{g}\left(K_{k, k}\right)=3$ for every $n \geq 2$. Hence the existence of an upper bound for the game chromatic number of the Cartesian product of graphs in terms of game chromatic numbers of factors would imply that there is a constant $n$ such that $\chi_{g}\left(K_{k, k} \square K_{m, m}\right) \leq n$ for any $k$ and $m$. However, we prove in this section that such a constant $n$ does not exist.

We need some more notation. Let $V\left(K_{k, k}\right)=V_{1} \cup V_{2}$ where $V_{i}$ are independent sets, and $V\left(K_{m, m}\right)=W_{1} \cup W_{2}$ where $W_{i}$ are independent sets. Vertices of $K_{k, k} \square K_{m, m}$ can be partitioned into four subsets of the form $V_{i} \times W_{j}$ of equal cardinality. We denote these 
subsets by $A, B, C$ and $D$. Let the notation be chosen in such a way that every vertex of $A$ is adjacent to $m$ vertices of $C$, to $k$ vertices of $B$, and to no vertex of $D$.

Vertices of $A$ that all have the same set of neighbors from $B$ form a so-called $A$ - $B$-fiber. Similarly we define $B$-A-fibers and all other $X$ - $Y$-fibers where $X$ and $Y$ are chosen as distinct elements from $\{A, B, C, D\}$. Note that there are $m$ vertices in an $A$-C-fiber $L$ and they have $m$ neighbors in $C$ that form a $C$-A-fiber. We call the latter a complementary $C$-A-fiber of $L$. Analogously we define other complementary $X$ - $Y$-fibers.

Theorem 4. Let $n$ be an arbitrary natural number. Then there exist natural numbers $k$ and $m$ such that $\chi_{g}\left(K_{k, k} \square K_{m, m}\right)>n$.

Proof. The game is played with $n$ colors in $G=K_{k, k} \square K_{m, m}$. For the time being we may assume that $k$ and $m$ can be as large as we need to ensure there are enough vertices available in $X$ - $Y$-fibers. The game begins by Alice coloring a vertex. Bob's goal is to force the existence of an uncolored vertex whose neighbors used all the $n$ colors from $\{1, \ldots, n\}$. Such a vertex will appear in the set $A$.

At a particular moment during the game we denote by $C V(G)$ the set of vertices which have already been colored. For $u \in C V(G)$ let $c(u)$ denote the color of $u$. For $L \subseteq V(G)$ we define $C V(L)=L \cap C V(G)$ and $c(L)=\{c(u) \mid u \in C V(L)\}$. If at a particular moment during the game $c(L)=\emptyset$, we will say that $L$ is empty.

We divide the strategy of Bob into two parts. In the first part he only colors vertices from $C$. The first part is further divided into consecutive phases. Each phase starts with Bob choosing an empty $C$-A-fiber $L$ whose complementary $A$ - $C$-fiber $L^{\prime}$ is also empty. Each phase then consists of $\lceil n / 2\rceil$ moves of Bob (and $\lfloor n / 2\rfloor$ moves made by Alice) in which Bob colors only the vertices from the $C$-A-fiber $L$ he had chosen at the beginning of the phase, no matter what Alice does. He is only restricted with the colors which Alice chooses in the complementary $A$ - $C$-fiber $L^{\prime}$ (notably, if Alice colors a vertex in $L^{\prime}$ by some color, then Bob cannot color vertices in $L$ by this color).

Consider a moment during the game, when a new phase begins, and with respect to this moment define $\mathcal{F}^{\prime}=\{c(L): L$ is a $C$-A-fiber with $|c(L)| \geq\lceil n / 2\rceil\}$, and $\mathcal{F}=\{X$ : $\left.X \subseteq Y, Y \in \mathcal{F}^{\prime},|X|=\lceil n / 2\rceil\right\}$. Now, consider the FS game of combinations with respect to $\mathcal{F}$ in which Bob is the avoider and Alice is the forcer. We have two possibilities: either the avoider has a winning strategy in this game or not (and in the latter case the forcer has a winning strategy). Clearly in the beginning of the game $\mathcal{F}$ is empty, and so the avoider (Bob) has a winning strategy.

Suppose that at the beginning of a phase the avoider (Bob) has a winning strategy in the FS game as described above. Then Bob picks one by one the integers (colors) from $[n]$ according to the winning strategy of the avoider and by these integers colors uncolored vertices in $L$. The moves of Alice in $L^{\prime}$ (that is the colors that she chooses in $L^{\prime}$ ) are considered as the moves of the forcer since these are precisely the colors that Bob cannot choose in $L$ during this phase. All moves of Alice that are not in $L^{\prime}$ are considered to be passes of the forcer in the FS game. Since Bob has a winning strategy, the combination of colors in $L$ that are chosen by Bob is distinct from all the combinations in $\mathcal{F}$. 
The second possibility happens after at most $\left(\begin{array}{c}n \\ \left\lceil\frac{n}{2}\right\rceil\end{array}\right)$ phases. Then the avoider no longer has a winning strategy in the FS game of combinations (with respect to $\mathcal{F}$, which is derived from combinations of colors in $C$-A-fibers as defined above). When this happens, Alice has a winning strategy as the forcer. This means that she could force Bob to use a $\lceil n / 2\rceil$ combination of colors (in a newly chosen $C$-A-fiber $L$ ) that is contained in $c(K)$ where $K$ is a $C$-A-fiber in $\mathcal{F}^{\prime}$ (and she can achieve this by playing only in the complementary $A$ - $C$-fiber $L^{\prime}$ of $\left.L\right)$. When this happens the first part of the game is over for Bob.

In the second part, Bob chooses an empty $B$ - $A$-fiber $M$ whose complementary $A$ - $B$ fiber $M^{\prime}$ is also empty. Again let $\mathcal{F}^{\prime}=\{c(L): L$ is a $C$-A-fiber with $|c(L)| \geq\lceil n / 2\rceil\}$, and $\mathcal{F}=\left\{X: X \subseteq Y, Y \in \mathcal{F}^{\prime},|X|=\lceil n / 2\rceil\right\}$, but now Bob plays as the forcer in the AS game of combinations with respect to $\mathcal{F}$. All moves of Bob are in $M$, and he colors the vertices of $M$ according to a winning strategy of the forcer in this game. Note that he considers only Alice's moves in $M^{\prime}$ as the moves in the AS game, and all other Alice's moves are considered as passes of the move (in particular, Alice passes the first move). Since forcer has a winning strategy in the AS game, the game ends after Bob colors $\lfloor n / 2\rfloor$ vertices in $M$. Note that the complement of colors with respect to $[n]$ which Bob used in $M$ is contained in $c(K) \in \mathcal{F}^{\prime}$ where $K$ is a $C$-A-fiber. We derive that in the neighborhood of the unique vertex $u$ in $M^{\prime} \cap K^{\prime}$ all $n$ colors appear (this in particular implies that the vertex $u \notin C V(G))$, and so Bob is the winner. If the game was played with less than $n$ colors, Bob can clearly use the same strategy, hence $\chi_{g}\left(K_{k, k} \square K_{m, m}\right)>n$, and the proof is complete.

We conclude this section with a word on the sizes of $K_{k, k}$ and $K_{m, m}$ that are needed in the above proof. Recall that the first part of the game consists of at most $\left(\begin{array}{c}n \\ \left\lceil\frac{n}{2}\right\rceil\end{array}\right)$ phases (this number is likely much smaller, and the exact number is related to the Question 2). Each phase takes $\left\lceil\frac{n}{2}\right\rceil$ Bob's moves, and if all moves of Alice are played in different fibers, we infer that

$$
k \geq\left\lceil\frac{n}{2}\right\rceil \cdot\left(\begin{array}{c}
n \\
\left\lceil\frac{n}{2}\right\rceil
\end{array}\right)+\left(\begin{array}{c}
n \\
\left\lceil\frac{n}{2}\right\rceil
\end{array}\right)+1
$$

ensures that the first part of the game can be performed (note that to the maximum number of moves of Alice we added the number of fibers chosen by Bob, and 1 because Alice has the first move). Hence the total number of moves in the first part of the game is at most $2\left\lceil\frac{n}{2}\right\rceil\left(\begin{array}{c}n \\ \left\lceil\frac{n}{2}\right\rceil\end{array}\right)+1$ and by letting

$$
m \geq 2\left\lceil\frac{n}{2}\right\rceil\left(\begin{array}{c}
n \\
\left\lceil\frac{n}{2}\right\rceil
\end{array}\right)+3 .
$$

we ensure that after Alice plays another move there still exists an empty $B$ - $A$-fiber $M$ whose complementary $A$ - $B$-fiber $M^{\prime}$ is empty too.

Thus the game chromatic number of a product $G \square H$ is not bounded by any function of game chromatic numbers of both factors. Is this still true if we look at some special 
Cartesian product graphs? In particular, Propositions 1, 2 and 3 suggest that this could be the case for prisms, that is the products $K_{2} \square G$. However, this is not true as the following example shows.

Let $G=v \cup\left(K_{n, n}-M\right)$, thus a single vertex $v$ and a $K_{n, n}$ without a perfect matching $M$. It is well-known that $\chi_{g}\left(K_{n, n}-M\right)=n$, however $\chi_{g}(G)=2$ since Alice can force Bob to make the first move in $K_{n, n}-M$. In prism $K_{2} \square G$ Bob can wait so that Alice makes the first move in the subgraph isomorphic to $K_{2} \square\left(K_{n, n}-M\right)$ and then just follows the strategy for $K_{n, n}-M$ in the same fiber as Alice. Note that there is no influence from the other fiber since Bob has a unique strategy for achieving $n$ colors in $K_{n, n}-M$. Thus $\chi_{g}\left(K_{2} \square G\right) \geq n$. Graph $G$ is not connected which could make this example less interesting. However by adding to $G$ an edge between $v$ and a vertex of $K_{n, n}-M$ we get a connected graph $H$ for which the same argument holds. It is easy to see that $\chi_{g}(H)=3$, but $\chi_{g}\left(K_{2} \square H\right) \geq n$ by the same strategy as in $G$.

On a positive side, the game chromatic number is bounded in the class of Cartesian products of trees. This follows from two results. The first is a bound of Dinski and Zhu $[7]$

$$
\chi_{g}(G) \leq a(G)(a(G)+1)
$$

where $G$ is an arbitrary graph, and $a(G)$ is the acyclic chromatic number of $G$, defined as the least number of colors needed for a proper vertex coloring of $G$ with no 2-colored cycles. Combining this with a result from [12] that $a\left(T_{1} \square T_{2}\right) \leq 3$ where $T_{i}$ are arbitrary trees, we get

$$
\chi_{g}\left(T_{1} \square T_{2}\right) \leq 12 .
$$

We wonder if one can improve this bound, so we ask the following.

Question 3. What is the smallest $k$ such that for any two trees $T_{1}$ and $T_{2}$

$$
\chi_{g}\left(T_{1} \square T_{2}\right) \leq k ?
$$

In particular, is $k<12$ ?

Combining the following inequality

$$
a\left(G_{1} \square G_{2}\right) \leq a\left(G_{1}\right) a\left(G_{2}\right),
$$

which holds for any pair of graphs $G_{1}$ and $G_{2}$, with the famous theorem of Borodin [5] that $a(G) \leq 5$ for every planar graph $G$ we infer that $a\left(G_{1} \square G_{2}\right) \leq 25$ if $G_{1}$ and $G_{2}$ are planar graphs. Hence by (1) we deduce that

$$
\chi_{g}\left(G_{1} \square G_{2}\right) \leq 650
$$

for every two planar graphs $G_{1}$ and $G_{2}$. This is rather not best possible.

Question 4. What is the smallest $k$ such that $\chi_{g}\left(G_{1} \square G_{2}\right) \leq k$ for any two planar graphs $G_{1}$ and $G_{2}$ ? 
The strategy based on acyclic coloring is not optimal for planar graphs; better results are obtained by the activation strategy (cf. [1]). So, it seems natural to expect that using activation will be more efficient also for the Cartesian product of planar graphs. Curiously, this idea cannot be successful, as we will see in the next section.

\section{Variations}

We start with the well-known marking game. Suppose that players do not possess colored pencils, but they still want to play a similar game. This leads to a related graph invariant called game coloring number. In this game Alice and Bob mark vertices, they alternate turns with Alice having the first move. Let $k$ be a natural number. The aim of Bob is similar as before: he wants to create a situation in which there is an unmarked vertex $x$ that has in its neighborhood $k$ vertices that are marked. Alice wants to prevent such a situation. Given a graph $G$ the smallest $k$ for which Alice has a winning strategy (that is, she can ensure that at each stage of the game every uncolored vertex has at most $k-1$ marked neighbors) is called the game coloring number of $G$, denoted $\operatorname{col}_{g}(G)$. Clearly, $\chi_{g}(G) \leq \operatorname{col}_{g}(G)$ for any graph $G$.

The concept is related to another graph invariant called coloring number that was introduced by Erdős and Hajnal [8]. Let $G$ be a graph and $L=\left(v_{1}, \ldots, v_{n}\right)$ a linear order of its vertices, where $n=|V(G)|$. The back degree $\operatorname{deg}_{d}\left(v_{i}\right)$ with respect to $L$ of a vertex $v_{i}$ is the number of neighbors $v_{j}$ of $v_{i}$ with $j<i$ (i.e. the neighbors that precede $v_{i}$ with respect to $\left.L\right)$. The back degree of a linear order $L$ is defined as the maximum back degree of a vertex with respect to $L$. The coloring number $\operatorname{col}(G)$ of a graph $G$ is the minimum integer $k$ such that $G$ has a linear order $L$ with back degree $k-1$. Clearly for any graph we have $\chi(G) \leq \operatorname{col}(G) \leq \Delta(G)+1$. Since the game coloring number is a back degree with respect to a (specific) linear order (obtained by Alice and Bob coloring $G$ using their optimal strategies), we get $\operatorname{col}(G) \leq \operatorname{col}_{g}(G)$. It is easy to see that $\operatorname{col}(G)=\operatorname{col}_{g}(G)=\Delta(G)+1$ for a regular graph $G$. The following recent result from [6] shows that the coloring number of the Cartesian product of graphs is bounded by a linear function of coloring numbers of factor graphs: for any graphs $G$ and $H$, we have $\operatorname{col}(G \square H) \leq \operatorname{col}(G)+\operatorname{col}(H)-1$.

Similarly as in the case of the chromatic number, the game variation of the coloring number does not enjoy such a property. Note that $\operatorname{col}_{g}\left(K_{n, n}\right)=n+1$ thus complete bipartite graphs cannot be used for the purposes of proving that the game coloring number of Cartesian products is not bounded by a function of game coloring numbers of factor graphs. However the class of Cartesian products of stars $K_{1, n}$ already serves this purpose for the case of game coloring number (but again not for the game chromatic number, as $\left.\chi_{g}\left(K_{1, n} \square K_{1, m}\right) \leq 12\right)$. Note that $\operatorname{col}_{g}\left(K_{1, n}\right)=2$.

Theorem 5. For any natural number $k$ there exist a natural number $n$ such that

$$
\operatorname{col}_{g}\left(K_{1, n} \square K_{1, n}\right)>k \text {. }
$$


The above result can be proved by similar methods as Theorem 4, but it also follows from a result of Kierstead and Trotter [14]. They proved that the game coloring number is not bounded in the class of subdivision graphs of $K_{n, n}$ (the graphs obtained from $K_{n, n}$ by subdividing all edges by single vertices). Note that the graph obtained from $K_{1, n} \square K_{1, n}$ by deletion of the vertex with the largest degree is isomorphic to the subdivided $K_{n, n}$. From this one can easily infer Theorem 5 .

Several variations of the coloring and marking game have appeared in the literature, and some of them have been arranged to work in the context of directed graphs, cf. [15]. We propose a few more, in particular in hypergraphs. They are related to some of our results and go along well with the product structure. This does not seem to be the case for the two main game invariants on graphs as the results of this paper show.

1. We start with the variation which is related to the marking game, and we call it vertex-edge marking game. Again two players play the game, and while the first player Alice marks vertices, Bob marks edges. The goal of Bob is to surround an unmarked vertex by as many marked edges as possible, while the goal of Alice is opposite; she wishes to keep the number of marked edges incident to an unmarked vertex as small as possible. The smallest $k$ for which Alice has a winning strategy in a graph $G$ (that is, she can keep the number of marked edges incident to any unmarked vertex less than $k$ ), will be denoted by $\operatorname{col}_{v e}(G)$.

Recalling again the result of Kierstead and Trotter [14] (or Theorem 5), one can prove in a similar way that $\operatorname{col}_{v e}\left(K_{n, n}\right)$ is not bounded by a constant.

Proposition 6. For any positive integer $k$ there exists $n$ such that $\operatorname{col}_{v e}\left(K_{n, n}\right)>k$.

We can also easily prove the following result.

Proposition 7. For any graphs $G$ and $H$,

$$
\operatorname{col}_{v e}(G \square H) \leq \operatorname{col}_{v e}(G)+\operatorname{col}_{v e}(H)-1
$$

and the bound is sharp.

Proof. Note that any move of Bob (which is marking of an edge) is made within a certain $G$-fiber or $H$-fiber. Hence what Alice must do each time is to respond within the very same fiber by the optimal move that exists in the corresponding factor graph. The upper bound clearly follows. The sharpness of the bound follows already from $\operatorname{col}_{v e}\left(K_{2}\right)=2$ and $\operatorname{col}_{v e}\left(K_{2} \square K_{2}\right)=3$.

2. Consider a generalization of the above game to hypergraphs, where Alice marks vertices and Bob marks (hyper)edges, and the goal of Bob is again to have an unmarked vertex included in as many marked edges as possible. This yields the hypergraph invariant which we also denote by $\operatorname{col}_{v e}$. Can we say something similar as in Proposition 7 for $\operatorname{col}_{v e}(G \times H)$ where $G$ and $H$ are hypergraphs? Recall that the product of hypergraphs $G$ and $H$ is the hypergraph $G \times H$ with $V(G) \times V(H)$ as the set of its vertices and $\{X \times Y: X \in E(G), Y \in E(H)\}$ as the set of its (hyper)edges. 
3. Finally consider a natural generalization of the coloring game for hypergraphs: Alice and Bob color vertices of a hypergraph $H$ and creating a monochromatic edge of $H$ is forbidden for either player. Goals of the players are the same as in the original game and the parameter $\chi_{g}(H)$ is defined in the same way. For instance, if $H$ is a hypergraph of cycles of a given graph $F$, we get the game of arboricity considered in [2].

Question 5. Is it true that $\chi_{g}(G \times H) \leq \min \left\{\chi_{g}(G), \chi_{g}(H)\right\}$ for arbitrary hypergraphs $G$ and $H$ ?

Acknowledgment. We wish to thank an anonymous referee for comments that resulted in a more transparent proof of the main theorem. This referee also pointed out that Theorem 5 was essentially proved by H. A. Kierstead and T. Trotter in [14].

\section{References}

[1] T. Bartnicki, J. Grytczuk, H. A. Kierstead, X. Zhu, The map coloring game, Amer. Math. Monthly, November, 2007.

[2] T. Bartnicki, J. Grytczuk, H. A. Kierstead, The game of arboricity, Discrete Math. 308 (2008) 1388-1393.

[3] J. Beck, Ramsey games, Discrete Math. 249 (2002) 3-30.

[4] J. Beck, Positional games, Combin. Probab. Comput. 14 (2005) 649-696.

[5] O. V. Borodin, On acyclic colorings of planar graphs, Discrete Math. 25 (1979) 211236.

[6] M. Borowiecki, S. Jendrol, D. Král, J. Miškuf, List coloring of Cartesian products of graphs, Discrete Math. 306 (2006) 1955-1958.

[7] T. Dinski, X. Zhu, Game chromatic number of graphs, Discrete Math. 196 (1999) 109-115.

[8] P. Erdős, A. Hajnal, On chromatic number of graphs and set-systems. Acta Math. Acad. Sci. Hungar 17 (1966) 61-99.

[9] A. S. Fraenkel, Combinatorial games: selected bibliography with a succinct gourmet introduction, Electron. J. Combin., Dynamic Survey 2 (2007) 78pp.

[10] D. Guan, X. Zhu, Game chromatic number of outer planar graphs. J. Graph Theory 30 (1999) 67-70.

[11] W. Imrich and S. Klavžar, Product graphs: Structure and Recognition (John Wiley \& Sons, New York, 2000).

[12] R. E. Jamison, G. L. Matthews, J. Villalpando, Acyclic colorings of products of trees. Inform. Process. Lett. 99 (2006) 7-12.

[13] H. A. Kierstead, T. Trotter, Planar graph coloring with an uncooperative partner, J. Graph Theory 18 (1994) 569-584. 
[14] H. A. Kierstead, T. Trotter, Competitive colorings of oriented graphs, Electron. J. Combin. 8 (2001) \#R12, 15pp.

[15] J. Nešetřil, E. Sopena, On the oriented game chromatic number, Electron. J. Combin. 8 (2001) \#R14, 13pp.

[16] J. Wu, X. Zhu, Lower bounds for the game colouring number of partial k-trees and planar graphs, manuscript 2003.

[17] X. Zhu, Game coloring number of pseudo partial k-trees. Discrete Math. 215 (2000) $245-262$.

[18] X. Zhu, Refined activation strategy for the marking game, J. Combin. Theory ser. B 98 (2008) 1-18. 